\title{
Application of renormalization to the dynamics of a particle in a infinite square-well potential driven by an external field
}

\author{
C. Chandre \\ Service de Physique Théorique, CEA Saclay, F-91191 Gif-sur-Yvette Cedex, France
}

\begin{abstract}
We analyze by a renormalization method, the dynamics of a particle in a infinite square-well potential driven by an external monochromatic field. This method set up for Hamiltonian systems with two degrees of freedom allows us to analyze precisely the stability of the trajectories of the particle as a function of the amplitude $\varepsilon$ of the external field. We compute numerical values of $\varepsilon$ for which the motion of the particle with frequency $\omega$ is broken and a transition to a chaotic behavior occurs. We obtain the critical function $\varepsilon_{c}(\omega)$ associated with this system as a function of the parameters such as the frequency of the field and the width of the potential.
\end{abstract}

PACS : 05.45.-a, 05.10.Cc, 05.45.Ac

\section{INTRODUCTION}

Due to the existence of as many conserved quantities as degrees of freedom, the trajectories of an integrable Hamiltonian system are confined to evolve on invariant rotational tori. On a given torus, the dynamics is regular, i.e. conjugate to a linear flow with frequency $\omega$ in action-angle coordinates. This regularity is broken by any small perturbation, and chaotic trajectories appear : The phase space of a Hamiltonian system close to integrable is in general a mixture of regular and chaotic motions. For Hamiltonian systems with two degrees of freedom, these invariant rotational tori, also called KAM (Kolmogorov-Arnold-Moser) tori, act as barriers in phase space.

For a given one parameter family of Hamiltonians $\left\{H_{\varepsilon}\right\}$ with $H_{\varepsilon=0}$ integrable and $\varepsilon$ the amplitude of the perturbation, it appears from numerical evidences that for $\varepsilon$ smaller than a critical value denoted $\varepsilon_{c}(\omega)$, there exists a KAM torus with frequency $\omega$, and this torus is broken for larger values by resonance or overlapping of resonances. The function $\omega \mapsto \varepsilon_{c}(\omega)$ is called the critical function associated with the one-parameter family of Hamiltonians $\left\{H_{\varepsilon}\right\}$. This function contains the information on the existence of invariant tori in phase space as the parameter $\varepsilon$ increases. In particular, for $\varepsilon>\sup _{\omega \in B} \varepsilon_{c}(\omega)$, there is no longer any KAM torus in the region of phase space corresponding to the set of frequencies $B$, and we have large scale stochasticity.

Renormalization methods have been defined and studied numerically for the analysis of stability of Hamiltonian systems with two degrees of freedom [1 6 . The aim is to describe the break-up of a given invariant torus. The idea is to set up a transformation that focuses on a specific region of phase space around the given torus. It acts as a microscope in phase space, looking at the system on smaller scales in phase space and on longer time scales. The complete renormalization method [2, 5.,6] is a tool to compute precisely the critical function $\varepsilon_{c}(\omega)$. It has been verified that for specific models (like a forced pendulum) the critical couplings obtained by renormalization coincide with other methods like Greene's residue criterion [7] or Laskar's frequency map analysis [8 [10].

The system we analyze is a particle of mass $m$ in an infinite square-well potential $V_{S Q}$ of width $2 a$ driven by an external monochromatic field with amplitude $\varepsilon$ and frequency $\Omega$. The Hamiltonian of this system with 1.5 degrees of freedom is the following :

$$
H(p, x, t)=\frac{p^{2}}{2 m}+V_{S Q}(x)+\varepsilon x \cos (\Omega t)
$$

where

$$
V_{S Q}(x)=0 \text { for }|x|<a \text { and } V_{S Q}(x)=+\infty \text { for }|x| \geq a,
$$

and $\varepsilon \geq 0$. This system has been studied in Refs. [11,12] by approximate renormalization methods. Without external field, i.e. for $\varepsilon=0$, the system is integrable and the motion is periodic with frequency $\omega=\sqrt{\frac{\pi^{2} E}{2 m a^{2}}}$ where $E$ denotes the energy of the system. For $\varepsilon \neq 0$, some of these regular motions disappear. In particular, there are resonances when the frequency of the external field $\Omega$ is commensurate with the frequency of the motion, i.e. when $\Omega$ is equal to $\frac{P}{Q} \omega$ ( $P$ and $Q$ are relatively prime integers). The interaction of these resonances breaks up some invariant tori (in the spirit of Chirikov's criterion 13,14]). The critical function $\varepsilon_{c}(\omega ; m, a, \Omega)$ is the critical value of the amplitude of the field for which the motion with frequency $\omega$ is broken. 
The aim of this paper is to apply renormalization to a specific model and to compute numerically $\varepsilon_{c}(\omega ; m, a, \Omega)$. We use this critical function to locate chaotic zones and to determine critical parameters for which large scale stochasticity occurs. We compare some of the results obtained by the renormalization transformation with the ones obtained by other existing methods such as Greene's residue criterion, in order to validate the results obtained by renormalization.

In Sec. II, we explicit the model and give some information on its dynamics. In Sec. III, we give a short description of the renormalization method, and in Sec. IV we compute numerically and analyze the critical function $\varepsilon_{c}(\omega ; m, a, \Omega)$.

\section{MODEL}

The external field induces resonances in the system when $\Omega=\frac{P}{Q} \omega$ where $\omega$ is the frequency of the unperturbed motion, $\Omega$ the one of the external field and $P, Q$ relatively prime integers. Due to the specific form of the interaction between the particle and the field, the largest resonances (i.e. of order of the amplitude $\varepsilon$ of the field) are obtained with $Q=1$ and $P$ odd. This can be seen by writing Hamiltonian (1.1) in action-angle variables [12] :

$$
H(A, \varphi, t)=\frac{\pi^{2}}{8 m a^{2}} A^{2}-\frac{4 \varepsilon a}{\pi^{2}} \sum_{\substack{n \in \mathbb{Z} \\ n \text { odd }}} \frac{1}{n^{2}} \cos (n \varphi-\Omega t) .
$$

There is a resonance when $n \dot{\varphi}=\Omega$, which corresponds to $n \omega=\Omega$ since $\dot{\varphi}=\frac{\partial H}{\partial A}$ is the frequency $\omega$ of the motion. This resonance will be denoted 1:n in what follows. Hamiltonian (2.1) can be mapped into a time-independent Hamiltonian with two degrees of freedom by considering $-\Omega t$ as a new angle variable :

$$
H\left(A_{1}, A_{2}, \varphi_{1}, \varphi_{2}\right)=\frac{\pi^{2}}{8 m a^{2}} A_{1}^{2}-\Omega A_{2}-\frac{4 \varepsilon a}{\pi^{2}} \sum_{n \text { odd }} \frac{1}{n^{2}} \cos \left(n \varphi_{1}+\varphi_{2}\right) .
$$

We rescale time by a factor $\Omega$, i.e. we multiply Hamiltonian $(2.2)$ by $1 / \Omega$. We notice that this rescaling of time changes the frequency of a quasiperiodic motion of Hamiltonian (2.2) by a factor $\Omega$. The new rescaled frequency is now $\omega / \Omega$. We rescale the action variables by replacing $H(\boldsymbol{A}, \boldsymbol{\varphi})$ by $\lambda^{-1} H(\boldsymbol{A} / \lambda, \boldsymbol{\varphi})$ with a factor $\lambda=\pi^{2} /\left(4 m a^{2} \Omega\right)$. We notice that the rescaling in the actions does not change the equations of motion. After this rescaling, Hamiltonian (2.2) is equal to

$$
H(\boldsymbol{A}, \boldsymbol{\varphi})=\frac{A_{1}^{2}}{2}-A_{2}-\varepsilon^{\prime} \sum_{n \text { odd }} \frac{1}{n^{2}} \cos \left(n \varphi_{1}+\varphi_{2}\right),
$$

where $\varepsilon^{\prime}$ is the dimensionless amplitude of the external field given by :

$$
\varepsilon^{\prime}=\frac{\varepsilon}{m a \Omega^{2}} \text {. }
$$

For $\varepsilon^{\prime}=0$, Hamiltonian (2.3) depends only on $\boldsymbol{A}=\left(A_{1}, A_{2}\right)$ and the equations of motion show that $A_{1}(t)$ and $A_{2}(t)$ are constant, and $\varphi_{1}(t)=\omega t+\varphi_{1,0}$ and $\varphi_{2}(t)=-t+\varphi_{2,0}$. The trajectories of the system (2.3) evolve on three-dimensional energy surfaces in the four-dimensional phase space, and for $\varepsilon^{\prime}=0$ the trajectories with frequency $\omega$ are confined to evolve on a two-dimensional torus (on the energy surface) with frequency vector $\boldsymbol{\omega}=(\omega,-1)$. For $\varepsilon^{\prime}>0$, this system has an infinite number of main resonances (given by the condition $n \dot{\varphi_{1}}+\dot{\varphi}_{2} \approx 0$ ) located around $A_{1}=1 / n$ where $n$ is an odd integer, accumulating at $A_{1}=0$. The width of the $n$-th resonance zone is approximately equal to $4 \sqrt{\varepsilon^{\prime}} / n$ [11]. For large values of $\varepsilon^{\prime}$ the torus with frequency vector $\boldsymbol{\omega}$ is broken by overlapping of resonances. In order to have an estimate of the critical value of $\varepsilon^{\prime}$ of the break-up of the invariant torus with frequency $\omega$, we apply Chirikov's criterion [13. For a torus with frequency $\omega$ located between the two primary resonances 1:n and 1:n+2, the overlapping is obtained when the sum of the two half-width of these two resonances is equal to their distance, i.e. for

$$
\varepsilon^{(c)}=\frac{1}{4(n+1)^{2}} .
$$

This value overestimates the critical values of the threshold of the break-up as it has been noticed in Ref. [11] for this model. A convenient way to compute the value of $\varepsilon^{\prime}$ for which the torus is broken is to set up a complete renormalization [2,5,6,9 in the spirit of Refs. [1,14. 


\section{RENORMALIZATION METHOD}

In this section, we give a description of the renormalization method we apply to the model described in the previous section.

First, we shift the actions such that the invariant torus with frequency $\omega$ is located at $A_{1}=0$ for the unperturbed Hamiltonian (for $\varepsilon^{\prime}=0$ ) : $A_{1}^{\prime}=A_{1}-\omega$ and $A_{2}^{\prime}=A_{2}$. Hamiltonian (2.3) is equal to :

$$
H\left(\boldsymbol{A}^{\prime}, \boldsymbol{\varphi}\right)=\boldsymbol{\omega} \cdot \boldsymbol{A}^{\prime}+\frac{1}{2}\left(\boldsymbol{\Omega} \cdot \boldsymbol{A}^{\prime}\right)^{2}-\varepsilon^{\prime} \sum_{\substack{\boldsymbol{\nu}=(n, 1) \\ n \text { odd }}} \frac{1}{n^{2}} \cos (\boldsymbol{\nu} \cdot \boldsymbol{\varphi})
$$

where $\boldsymbol{\Omega}=(1,0)$ and $\boldsymbol{\omega}=(\boldsymbol{\omega},-1)$.

The renormalization transformation is a map $H^{\prime}=\mathcal{R}(H)$ acting within the family of Hamiltonians of the form :

$$
H(\boldsymbol{A}, \boldsymbol{\varphi})=\boldsymbol{\omega} \cdot \boldsymbol{A}+V(\boldsymbol{\Omega} \cdot \boldsymbol{A}, \boldsymbol{\varphi})
$$

and Hamiltonian (3.1) is an element of this family. Furthermore Hamiltonian (3.1) is quadratic in the actions; this feature is useful for a simplification of the implementation of the transformation (see Step 4 below) [5]. However, a similar version of the transformation (obtained by slightly changing the way Step 4 is implemented) can be defined and studied numerically [6, 10].

The transformation $\mathcal{R}$ is based on the continued fraction expansion of $\omega$ :

$$
\omega=\frac{1}{a_{0}+\frac{1}{a_{1}+\cdots}} \equiv\left[a_{0}, a_{1}, \ldots\right] .
$$

The best rational approximates of $\omega$ are given by the truncations of its continued fraction expansion :

$$
\frac{p_{k}}{q_{k}}=\left[a_{0}, a_{1}, \ldots, a_{k}=\infty\right]
$$

The corresponding periodic orbits with frequency vectors $\left(p_{k} / q_{k},-1\right)$ which are orthogonal to the modes $\boldsymbol{\nu}_{k}=\left(q_{k}, p_{k}\right)$ $\left(\boldsymbol{\nu}_{k}\right.$ is called "resonance" and is also denoted $p_{k}: q_{k}$ in what follows) accumulate at the invariant torus with frequency vector $\boldsymbol{\omega}=(\boldsymbol{\omega},-1)$. This family of periodic orbits satisfies the following relations : $\left|\boldsymbol{\omega} \cdot \boldsymbol{\nu}_{k+1}\right|<\left|\boldsymbol{\omega} \cdot \boldsymbol{\nu}_{k}\right|$ and $\lim _{k \rightarrow \infty}\left|\boldsymbol{\omega} \cdot \boldsymbol{\nu}_{k}\right|=0$, and $\boldsymbol{\nu}_{k}$ is given by the following equation :

$$
\boldsymbol{\nu}_{k}=N_{a_{0}} \cdots N_{a_{k-1}} \boldsymbol{\nu}_{0}
$$

where $\boldsymbol{\nu}_{0}=(1,0)$ and $N_{a_{i}}$ denotes the matrix

$$
N_{a_{i}}=\left(\begin{array}{cc}
a_{i} & 1 \\
1 & 0
\end{array}\right)
$$

The set of the Fourier modes of the perturbation with frequency vectors $\boldsymbol{\nu}_{k}$ leads to the divergence of perturbation expansions since the small denominators which are equal to $\boldsymbol{\omega} \cdot \boldsymbol{\nu}_{k}$ tend to zero as $k$ increases. The renormalization transformation deals with the modes $\boldsymbol{\nu}_{k}$ specifically by non-perturbative techniques.

The transformation $\mathcal{R}$ is composed by four steps :

(1) A shift of resonances constructed from the condition $\boldsymbol{\nu}_{1} \mapsto \boldsymbol{\nu}_{0}$ : we impose that $\cos \left[\left(a_{0}, 1\right) \cdot \boldsymbol{\varphi}\right]=\cos [(1,0)$. $\left.\varphi^{\prime}\right]$ where $a_{0}=\left[\omega^{-1}\right]$ is the integer part of $\omega^{-1}$. This change of coordinates is performed by a linear canonical transformation

$$
(\boldsymbol{A}, \boldsymbol{\varphi}) \mapsto\left(\boldsymbol{A}^{\prime}, \boldsymbol{\varphi}^{\prime}\right)=\left(N_{a_{0}}^{-1} \boldsymbol{A}, N_{a_{0}} \boldsymbol{\varphi}\right)
$$

which is generated by $F\left(\boldsymbol{A}^{\prime}, \boldsymbol{\varphi}\right)=N_{a_{0}} \boldsymbol{A}^{\prime} \cdot \boldsymbol{\varphi}$. The Hamiltonian expressed in the new coordinates becomes

$$
\begin{aligned}
H^{\prime}\left(\boldsymbol{A}^{\prime}, \boldsymbol{\varphi}^{\prime}\right) & =H(\boldsymbol{A}, \boldsymbol{\varphi})=\boldsymbol{\omega} \cdot \boldsymbol{A}+V(\boldsymbol{\Omega} \cdot \boldsymbol{A}, \boldsymbol{\varphi}), \\
& =\boldsymbol{\omega} \cdot N_{a_{0}} \boldsymbol{A}^{\prime}+V\left(\boldsymbol{\Omega} \cdot N_{a_{0}} \boldsymbol{A}^{\prime}, N_{a_{0}}^{-1} \boldsymbol{\varphi}^{\prime}\right), \\
& =N_{a_{0}} \boldsymbol{\omega} \cdot \boldsymbol{A}^{\prime}+V\left(N_{a_{0}} \boldsymbol{\Omega} \cdot \boldsymbol{A}^{\prime}, N_{a_{0}}^{-1} \boldsymbol{\varphi}^{\prime}\right) .
\end{aligned}
$$

Thus the new frequency vector is equal to $N_{a_{0}} \boldsymbol{\omega}=-\boldsymbol{\omega} \boldsymbol{\omega}^{\prime}$ where $\boldsymbol{\omega}^{\prime}=\left(\boldsymbol{\omega}^{\prime},-1\right)$ and $\boldsymbol{\omega}^{\prime}$ is the image of $\boldsymbol{\omega}$ by the Gauss map

$$
\omega \mapsto \omega^{\prime}=\omega^{-1}-\left[\omega^{-1}\right]
$$


This map corresponds to a shift to the left of the entries in the continued fraction expansion of the frequency

$$
\omega=\left[a_{0}, a_{1}, a_{2}, \ldots\right] \mapsto \omega^{\prime}=\left[a_{1}, a_{2}, a_{3}, \ldots\right] .
$$

The main effect of Step 1 is to change the frequency vectors of the Fourier modes of the perturbation according to the map $\boldsymbol{\nu} \mapsto N_{a_{0}}^{-1} \boldsymbol{\nu}$.

(2) We rescale the energy by a factor $\omega^{-1}$ (or equivalently time by a factor $\omega$ ), i.e. we multiply the Hamiltonian by $\omega^{-1}$, and we change the sign of both phase space coordinates $(\boldsymbol{A}, \boldsymbol{\varphi}) \mapsto(-\boldsymbol{A},-\boldsymbol{\varphi})$, in order to have $\boldsymbol{\omega}^{\prime}$ as the new frequency vector, i.e. such that the linear term in the actions $N_{a_{0}} \boldsymbol{\omega} \cdot \boldsymbol{A}=-\boldsymbol{\omega} \boldsymbol{\omega}^{\prime} \cdot \boldsymbol{A}$ is rescaled into a term of the form $\boldsymbol{\omega}^{\prime} \cdot \boldsymbol{A}$. Moreover, $\boldsymbol{\Omega}=(1, \alpha)$ is changed into $N_{a_{0}} \boldsymbol{\Omega}=\left(a_{0}+\alpha, 1\right)=\left(a_{0}+\alpha\right) \boldsymbol{\Omega}^{\prime}$ with $\boldsymbol{\Omega}^{\prime}=\left(1, \alpha^{\prime}\right)=\left(1,\left(a_{0}+\alpha\right)^{-1}\right)$ since the normalization condition we use is that the first component of $\boldsymbol{\Omega}$ is equal to one. The map $\alpha \mapsto\left(a_{0}+\alpha\right)^{-1}$ is the inverse of the Gauss map (3.4), in the sense that if $\alpha=\left[b_{0}, b_{1}, \ldots\right]$ then $\alpha^{\prime}=\left[a_{0}, b_{0}, b_{1}, \ldots\right]$. If we define

$$
[\alpha \mid \omega]=\left[\ldots, b_{2}, b_{1}, b_{0} \mid a_{0}, a_{1}, a_{2}, \ldots\right],
$$

The map $[\alpha \mid \omega] \mapsto\left[\alpha^{\prime} \mid \omega^{\prime}\right]$ corresponds to a two-sided Bernoulli shift :

$$
\left[\ldots, b_{2}, b_{1}, b_{0} \mid a_{0}, a_{1}, a_{2}, \ldots\right] \mapsto\left[\ldots, b_{2}, b_{1}, b_{0}, a_{0} \mid a_{1}, a_{2}, \ldots\right] .
$$

Since $\omega<1$, the effect of the rescaling of time is that the trajectories of the rescaled Hamiltonian correspond to the ones of the initial Hamiltonian on a longer time scale.

(3) We perform a rescaling of the actions : $H$ is changed into

$$
H^{\prime}(\boldsymbol{A}, \boldsymbol{\varphi})=\lambda H\left(\frac{\boldsymbol{A}}{\lambda}, \boldsymbol{\varphi}\right),
$$

with $\lambda=\lambda(H)$ such that the mean value of the quadratic term of $H^{\prime}$ in the variable $\boldsymbol{\Omega}^{\prime} \cdot \boldsymbol{A}$, is equal to $\left(\boldsymbol{\Omega}^{\prime} \cdot \boldsymbol{A}\right)^{2} / 2$ :

$$
\lambda=2 \omega^{-1}\left(a_{0}+\alpha\right)^{2}\left\langle V^{(2)}\right\rangle,
$$

where $V^{(2)}$ denotes the coefficient of the quadratic term of $V(\boldsymbol{\Omega} \cdot \boldsymbol{A}, \boldsymbol{\varphi})=H-\boldsymbol{\omega} \cdot \boldsymbol{A}$ in the variable $\boldsymbol{\Omega} \cdot \boldsymbol{A}$, and $\left\langle V^{(2)}\right\rangle$ denotes its mean value on $\left[0,2 \pi\left[^{2}\right.\right.$. Since in general $\left\langle V^{(2)}\right\rangle$ is close to $1 / 2$, the rescaling coefficient (3.5) is larger than one. Thus the rescaling in the actions corresponds to a focus in phase space around the invariant torus with frequency $\boldsymbol{\omega}^{\prime}$ located approximately at $\boldsymbol{\Omega}^{\prime} \cdot \boldsymbol{A} \approx 0$.

After these three steps, $H$ is changed into

$$
H^{\prime}(\boldsymbol{A}, \boldsymbol{\varphi})=\boldsymbol{\omega}^{\prime} \cdot \boldsymbol{A}+2 \omega^{-2}\left(a_{0}+\alpha\right)^{2}\left\langle V^{(2)}\right\rangle V\left(-\frac{1}{2 \omega^{-1}\left(a_{0}+\alpha\right)\left\langle V^{(2)}\right\rangle} \boldsymbol{\Omega}^{\prime} \cdot \boldsymbol{A},-N_{a_{0}}^{-1} \boldsymbol{\varphi}\right) .
$$

(4) The fourth step is a canonical transformation that eliminates the non-resonant part (denoted $I^{-}$) of the perturbation of $H^{\prime}$.

The choice of the part of the perturbation which has to be considered resonant or non-resonant is somewhat arbitrary. The set of non-resonant modes contains the modes of the perturbation which are sufficiently far from the resonances $\left\{\boldsymbol{\nu}_{k}\right\}$ in order to avoid small denominator problems during the elimination process. A convenient choice concerning the non-resonant modes is the set $I^{-}$of integer vectors $\boldsymbol{\nu}=\left(\nu_{1}, \nu_{2}\right)$ such that $\left|\nu_{2}\right|>\left|\nu_{1}\right|$ :

$$
I^{-}=\left\{\boldsymbol{\nu}=\left(\nu_{1}, \nu_{2}\right) \in \mathbb{Z}^{2}|| \nu_{2}|>| \nu_{1} \mid\right\} .
$$

We notice that Eq. (3.3) defining $\boldsymbol{\nu}_{k}=\left(q_{k}, p_{k}\right)$ shows that $p_{k} \leq q_{k}$ for $k \geq 0$. Consequently, the resonances do not belong to $I^{-}$. At each iteration of the transformation, the frequency vector of the considered torus changes (since we perform unimodular transformations). We have chosen a unique region $I^{-}$such that it does not contain any of the resonance lines $\boldsymbol{\omega} \cdot \boldsymbol{\nu}=0$ for all $\boldsymbol{\omega} \in] 0,1[$.

From the form of the eigenvectors of $N_{a_{i}}$, we can see that each vector $\boldsymbol{\nu} \in \mathbb{Z}^{2} \backslash\{\mathbf{0}\}$ is mapped into $I^{-}$after a sufficient number of iterations of the matrices $N_{a_{i}}$ (the eigenvector of $N_{a_{i}}^{-1}$ associated with the eigenvalue of modulus larger than one points into $I^{-}$). In other terms, each resonant mode becomes non-resonant at a sufficiently smaller scale in phase space. We notice that $\mathbf{0}$ is not an element of $I^{-}$, i.e. it is resonant.

Since the initial Hamiltonian (2.3) is quadratic in the actions, the renormalization for quadratic Hamiltonians defined in Refs. [5,9] is well-suited for this problem. We define more precisely Step 4 for the following Hamiltonians :

$$
H(\boldsymbol{A}, \boldsymbol{\varphi})=\boldsymbol{\omega} \cdot \boldsymbol{A}+m(\boldsymbol{\varphi})(\boldsymbol{\Omega} \cdot \boldsymbol{A})^{2}+g(\boldsymbol{\varphi}) \boldsymbol{\Omega} \cdot \boldsymbol{A}+f(\boldsymbol{\varphi}),
$$


where $m, g$ and $f$ are scalar functions of the angles and $\langle m\rangle \neq 0$. We eliminate completely the non-resonant modes of $g$ and $f$ by a canonical transformation connected to the identity, which is defined by iterating KAM-type transformations. The KAM iterations we perform (by Lie transformations) are generated by functions that are linear in the actions. The Hamiltonian expressed in the new coordinates is again quadratic in the actions. Thus this type of transformation allows us to remain quadratic at each step of the transformation 15. One iteration $\mathcal{U}_{S}$ of the KAM transformation reduces the non-resonant modes of $f$ and $g$, from order $\varepsilon$ to $\varepsilon^{2}$. The transformation that eliminates completely the non-resonant part is defined in the following way :

$$
H^{\prime}=H \circ \mathcal{U}_{H}, \text { where } \mathcal{U}_{H}=\mathcal{U}_{S_{1}} \circ \mathcal{U}_{S_{2}} \circ \cdots \mathcal{U}_{S_{n}} \circ \cdots,
$$

where the purpose of $\mathcal{U}_{S_{n}}$ is to reduce the non-resonant part of $f$ and $g$ from order $\varepsilon^{2^{n-1}}$ to $\varepsilon^{2^{n}}$, such that $\mathbb{I}^{-} f^{\prime}=\mathbb{I}^{-} g^{\prime}=$ 0 , where $\mathbb{I}^{-} f^{\prime}$ denotes the non-resonant part of the constant term in the actions of $H^{\prime}$, i.e. $\mathbb{I}^{-} f^{\prime}=\sum_{\boldsymbol{\nu} \in I^{-}} f_{\boldsymbol{\nu}}^{\prime} e^{i \boldsymbol{\nu} \cdot \boldsymbol{\varphi}}$. For the explicit equations of this part of the transformation, we refer to Refs. [5,16].

In summary, the renormalization transformation acts in the following way : First, some of the resonant modes are turned non-resonant by a rescaling of phase space that changes the frequency of the torus according to the Gauss map (3.4). Then an iteration of a KAM-type transformation eliminates the non-resonant modes (by slightly changing the resonant ones).

The renormalization transformation is a map of Fourier coefficients : If

$$
H(\boldsymbol{A}, \boldsymbol{\varphi})=\boldsymbol{\omega} \cdot \boldsymbol{A}+\sum_{k, \boldsymbol{\nu}} H_{k, \boldsymbol{\nu}}(\boldsymbol{\Omega} \cdot \boldsymbol{A})^{k} e^{i \boldsymbol{\nu} \cdot \boldsymbol{\varphi}},
$$

then $H^{\prime}=\mathcal{R}(\mathcal{H})$ becomes $\left\{H_{k, \boldsymbol{\nu}}^{\prime}\right\}=\mathcal{R}\left(\left\{H_{k, \boldsymbol{\nu}}\right\}\right)$ where $k=0,1,2$ in our case. The properties of $\mathcal{R}$ are obtained by iterating this map. In order to analyze it numerically, we truncate this map, i.e. we truncate the Fourier series by considering frequency vectors $\boldsymbol{\nu}=\left(\nu_{1}, \nu_{2}\right)$ such that $\max _{i=1,2}\left|\nu_{i}\right| \leq L$. We observe the convergence of the properties of the renormalization transformation as $L$ grows.

\section{CRITICAL FUNCTION OF THE MODEL}

\section{A. Critical function $\varepsilon_{c}^{\prime}(\omega)$}

The aim is to obtain the value $\varepsilon_{c}^{\prime}(\omega)$ for which the invariant torus with frequency $\omega$ exists for $\varepsilon^{\prime}<\varepsilon_{c}^{\prime}(\omega)$ and is broken for larger values.

If $\omega$ satisfies a Diophantine condition, the KAM theorem 17 ensures the persistence of an invariant torus with frequency $\omega$ for the system we consider, i.e. $\varepsilon_{c}^{\prime}(\omega)>0$. If $\omega$ is rational, a resonance breaks up the torus, i.e. $\varepsilon_{c}^{\prime}(\omega)=0$. Moreover, this function is symmetric : $\varepsilon_{c}^{\prime}(-\omega)=\varepsilon_{c}^{\prime}(\omega)$. This symmetry comes from the fact that the canonical transformation $\left(A_{1}, \varphi_{1}, A_{2}, \varphi_{2}\right) \mapsto\left(-A_{1},-\varphi_{1}, A_{2}, \varphi_{2}\right)$ only changes the frequency $\omega$ into $-\omega$ in Hamiltonian (2.3).

The critical function $\varepsilon_{c}^{\prime}(\omega)$ is determined by looking at the iterates of the renormalization transformation $\mathcal{R}$ described above, i.e. it is defined by the following equations :

$$
\begin{aligned}
& \mathcal{R}^{n} H_{\varepsilon^{\prime}} \underset{n \rightarrow \infty}{\rightarrow} H_{0}(\boldsymbol{A})=\boldsymbol{\omega} \cdot \boldsymbol{A}+\frac{1}{2}(\boldsymbol{\Omega} \cdot \boldsymbol{A})^{2} \quad \text { for } \varepsilon^{\prime}<\varepsilon_{c}^{\prime}(\omega), \\
& \mathcal{R}^{n} H_{\varepsilon^{\prime}} \underset{n \rightarrow \infty}{\rightarrow} \infty \quad \text { for } \varepsilon^{\prime}>\varepsilon_{c}^{\prime}(\omega),
\end{aligned}
$$

where $H_{\varepsilon^{\prime}}$ is Hamiltonian (3.1). Numerical work shows that Eqs.(4.1)-(4.2) define uniquely $\varepsilon_{c}^{\prime}(\omega)$, i.e. the renormalization has two main domains : one where the iterations of the transformation converge to an integrable Hamiltonian $H_{0}$ - this domain is conjectured to be the set of Hamiltonians that have a smooth invariant torus with frequency $\omega$ - and a domain where the iterations diverge, and this domain is conjectured to be the set of Hamiltonians that do not have an invariant torus with the frequency $\omega$. For $\varepsilon^{\prime}=\varepsilon_{c}^{\prime}(\omega)$, it was shown numerically that the iterations converge to a strange chaotic attractor containing all the relevant information on critical tori (i.e. at the threshold of the break-up) [9]. This means that we conjecture that the critical thresholds obtained by the complete renormalization coincide (up to numerical precision) with the thresholds of the break-up of invariant tori. In order to give support to this conjecture, we compare the values we obtain with another independent method, Greene's residue criterion. This method consists in analyzing the stability of nearby periodic orbits (for instance, the ones given by the truncations of the continued fraction expansion of the frequency of the torus). For rigorous results on Greene's criterion, we refer to Refs. [18, 19$]$. 
Figure 11 shows the value of $\varepsilon_{c}^{\prime}(\omega)$ for $\left.\omega \in\right] 0,1[$ determined by the renormalization method with $L=20$. A first remark is that the last invariant torus is not the golden mean one as it is the case for the standard map and for Escande's paradigm Hamiltonian [9] : The torus with frequency $\gamma=(\sqrt{5}-1) / 2=[1,1,1, \ldots]$ is broken for $\varepsilon_{c}^{\prime}(\gamma) \approx 0.02995$ whereas for instance the torus with frequency $\omega_{1}=(\gamma+3) / 5=[1,2,1,1,1, \ldots]$ persists until $\varepsilon_{c}^{\prime}\left(\omega_{1}\right) \approx 0.03163$. These two values coincide up to numerical precision with the ones obtained by Greene's residue criterion $: \varepsilon_{c}^{(G)}(\gamma) \approx 0.0299$ and $\varepsilon_{c}^{(G)}\left(\omega_{1}\right) \approx 0.0316$.

The last KAM torus is broken for $\varepsilon^{*} \approx 0.03334$. Its frequency is equal to $\omega_{2} \approx 0.6976$. The critical threshold of the break-up of this torus with frequency $\omega_{2}$ obtained by Greene's residue criterion coincides with $\varepsilon^{*}$ up to numerical precision : $\varepsilon_{c}^{(G)}\left(\omega_{2}\right) \approx 0.0333$. For $\varepsilon>\varepsilon^{*}$, there are no invariant tori left, and large scale stochasticity occurs : trajectories can go from the resonance 1:1 (located at $A_{1}=1$ ) to the resonance 0:1 (located at $A_{1}=0$ ).

Between two neighboring main resonances 1:n and 1:n+2, quasiperiodic motion with frequency $\omega \in] 1 /(n+2), 1 / n[$ can occur for small $\varepsilon^{\prime}$. If $\varepsilon^{\prime}$ is greater than some value $\varepsilon_{n}$, there is no longer any quasiperiodic motion in between these two resonances (and some chaotic trajectories can go from one resonance to the other). For $n=1$, this value is equal to $\varepsilon^{*}$ for which all rotational invariant tori are broken since the last invariant torus to break-up is located between the resonances 1:1 and 1:3. We apply Chirikov's criterion in order to have an estimate of $\varepsilon_{n}$ [see Eq.(2.5)] :

$$
\varepsilon_{n}^{(c)}=\frac{1}{4(n+1)^{2}} .
$$

For the largest one $n=1$, this value is equal to 0.0625 which is approximately twice the value obtained by renormalization. Escande's approximate renormalization gives 0.0352 as the critical amplitude of the field for the last invariant torus, which is close to the value determined by the complete renormalization method. This feature is expected to be true for quadratic Hamiltonians in the actions as it was pointed out in Refs. 20,21]. In Ref. [22], it has been noticed that the approximate renormalization usually slightly overestimates the real critical value.

Lin and Reichl 111] have developed a method adapted to the specific model (1.1) to compute the critical amplitudes $\varepsilon_{n}$. This method is based on the fact that as soon as the last invariant torus is broken, some trajectories starting near one of the main resonance (say 1:n) can approach the other main resonance 1:n+2. Since the diffusion of these trajectories can be very slow (due in particular to the resonances of low order between two main neighboring resonances, and in particular in the region of phase space where the last KAM torus breaks up when the parameter $\varepsilon^{\prime}$ is close to its critical value), the values they obtained overestimate the ones obtained by renormalization. For instance, between 1:1 and 1:3 the critical value they obtained is approximately $0.037 \pm 0.001$.

For $n=3$, the estimate obtained by Chirikov's criterion is $\varepsilon_{3}^{(c)} \approx 0.156$, Escande-Doveil's renormalization gives $\varepsilon_{3} \approx 0.0080$, whereas Lin and Reichl obtained $\varepsilon_{3} \approx 0.0081 \pm 0.0003$. The approximate value of $\varepsilon_{3}$ obtained by the complete renormalization is $\varepsilon_{3} \approx 0.0068$. Again, the same comments apply to this case : Lin and Reichl's method overestimates the value obtained by renormalization because of the very slow diffusion of the trajectories between the resonances 1:3 and 1:5 for critical or near-critical values of the parameter $\varepsilon^{\prime}$.

\section{B. Critical function $\varepsilon_{c}(\omega ; m, a, \Omega)$}

We have studied the critical function $\varepsilon_{c}^{\prime}(\omega)$ of Hamiltonian (2.3). This rescaled Hamiltonian is equivalent to the initial Hamiltonian (2.2) with $m a \Omega^{2}=1$ and in particular with $a=1, m=1$ and $\Omega=1$. For other values of $a, m$, and $\Omega$, the critical function $\varepsilon_{c}(\omega ; m, a, \Omega)$ is equal to

$$
\varepsilon_{c}(\omega ; m, a, \Omega)=m a \Omega^{2} \varepsilon_{c}^{\prime}\left(\frac{\omega}{\Omega}\right),
$$

according to Eq. (2.4). We notice that the argument of $\varepsilon_{c}^{\prime}$ is the rescaled frequency $\omega / \Omega$ since time has been rescaled by a factor $\Omega$. Thus the critical function varies like the square of the frequency of the field. In particular, the largest value of the parameter for which an invariant torus persists between the resonances 1:n and 1:n+2 varies like:

$$
\varepsilon_{n}(\Omega)=\Omega^{2} \varepsilon_{n}(\Omega=1),
$$

which is consistent with the numerical results found in Ref. [11]. This feature can be generalized to the following one-parameter family of Hamiltonians :

$$
H(p, x, t)=\frac{p^{2}}{2 m}+V_{S Q}(x)+\varepsilon \sum_{i} f_{i}(x) g_{i}(\Omega t),
$$


where $g_{i}$ are $2 \pi$-periodic functions. This can be seen by rescaling time by a factor $\Omega$

$$
H^{\prime}\left(p, x, t^{\prime}\right)=\frac{1}{\Omega} H\left(p, x, \frac{t^{\prime}}{\Omega}\right),
$$

and the actions by a factor $1 / \Omega$

$$
H^{\prime \prime}\left(p^{\prime}, x, t^{\prime}\right)=\frac{1}{\Omega} H^{\prime}\left(\Omega p^{\prime}, x, t^{\prime}\right) .
$$

The fact that the critical coupling $\varepsilon_{c}(\omega ; m, a, \Omega)$ is proportional to $m$ is general for a particle in an infinite square-well potential driven by a perturbation depending on $x$ and periodically on $t$. This is obtained by rescaling the momentum by a factor $1 / m$ of the Hamiltonian :

$$
H(p, x, t)=\frac{p^{2}}{2 m}+V_{S Q}(x)+\varepsilon V(x, t) .
$$

From the fact that the interaction with the field is proportional to $x$ (the interaction is of the form $\varepsilon x f(\Omega t)$ where $f$ is $2 \pi$-periodic), the critical function $\varepsilon_{c}(\omega ; m, a, \Omega)$ is expected to be proportional to $a$.

\section{CONCLUSION}

We have defined and studied numerically a renormalization transformation for a system of a particle in an infinite square well potential driven by an external monochromatic field in order to determine the critical thresholds of the break-up of invariant tori. Renormalization allows to obtain very precise information on the stability of Hamiltonian systems with two degrees of freedom, and in particular it allows to determine what kind of stable motions remains as a function of the amplitude of the perturbation. We have checked that for some specific frequencies, the thresholds obtained by renormalization coincide with the ones obtained by Greene's residue criterion.

We have chosen to study a particle in an infinite square well potential driven by an external field because of its simplicity, but the renormalization methods are very general and can be applied directly to other types of Hamiltonian systems with two effective degrees of freedom.

\section{ACKNOWLEDGMENTS}

The author acknowledges useful discussions with G. Benfatto, A. Celletti, H.R. Jauslin, H. Koch, J. Laskar and R.S. MacKay.

[1] D.F. Escande and F. Doveil, J. Stat. Phys. 26, 257 (1981).

[2] H. Koch, Erg. Theor. Dyn. Syst. 19, 475 (1999).

[3] M. Govin, C. Chandre, and H.R. Jauslin, Phys. Rev. Lett. 79, 3881 (1997).

[4] C. Chandre, M. Govin, and H.R. Jauslin, Phys. Rev. E 57, 1536 (1998).

[5] C. Chandre, M. Govin, H.R. Jauslin, and H. Koch, Phys. Rev. E 57, 6612 (1998).

[6] J.J. Abad, H. Koch, and P. Wittwer, Nonlinearity 11, 1185 (1998).

[7] J.M. Greene, J. Math. Phys. 20, 1183 (1979).

[8] J. Laskar, in Hamiltonian Systems with Three or More Degrees of Freedom, NATO ASI Series, edited by C. Simó (Kluwer Academic Publishers, Dordrecht, 1999), pp. $134-150$.

[9] C. Chandre and H.R. Jauslin, Phys. Rev. E 61, 1320 (2000).

[10] C. Chandre, J. Laskar, G. Benfatto, and H.R. Jauslin, (unpublished).

[11] W.A. Lin and L.E. Reichl, Physica 19D, 145 (1986).

[12] L.E. Reichl, The Transition to Chaos in Conservative Classical Systems: Quantum Manifestations (Springer-Verlag, New York, 1992).

[13] B.V. Chirikov, Phys. Rep. 52, 263 (1979). 
[14] D.F. Escande, Phys. Rep. 121, 165 (1985).

[15] W. Thirring, A Course in Mathematical Physics I: Classical Dynamical Systems (Springer-Verlag, Berlin, 1992).

[16] C. Chandre and H.R. Jauslin, in Mathematical Results in Statistical Mechanics, edited by S. Miracle-Solé, J. Ruiz, and V. Zagrebnov (World Scientific, Singapore, 1999).

[17] G. Gallavotti, in Scaling and Self-Similarity in Physics, Vol. 7 of PPh., edited by J. Fröhlich (Birkäuser, Boston, 1983), pp. 359-426.

[18] R.S. MacKay, Nonlinearity 5, 161 (1992).

[19] C. Falcolini and R. de la Llave, J. Stat. Phys. 67, 609 (1992).

[20] L.E. Reichl and W.M. Zheng, Phys. Rev. A 30, 1068 (1984).

[21] W.A. Lin and L.E. Reichl, Phys. Rev. A 31, 1136 (1985).

[22] C. Chandre, H.R. Jauslin, and G. Benfatto, J. Stat. Phys. 94, 241 (1999).

FIG. 1. Critical function of Hamiltonian system (3.1)

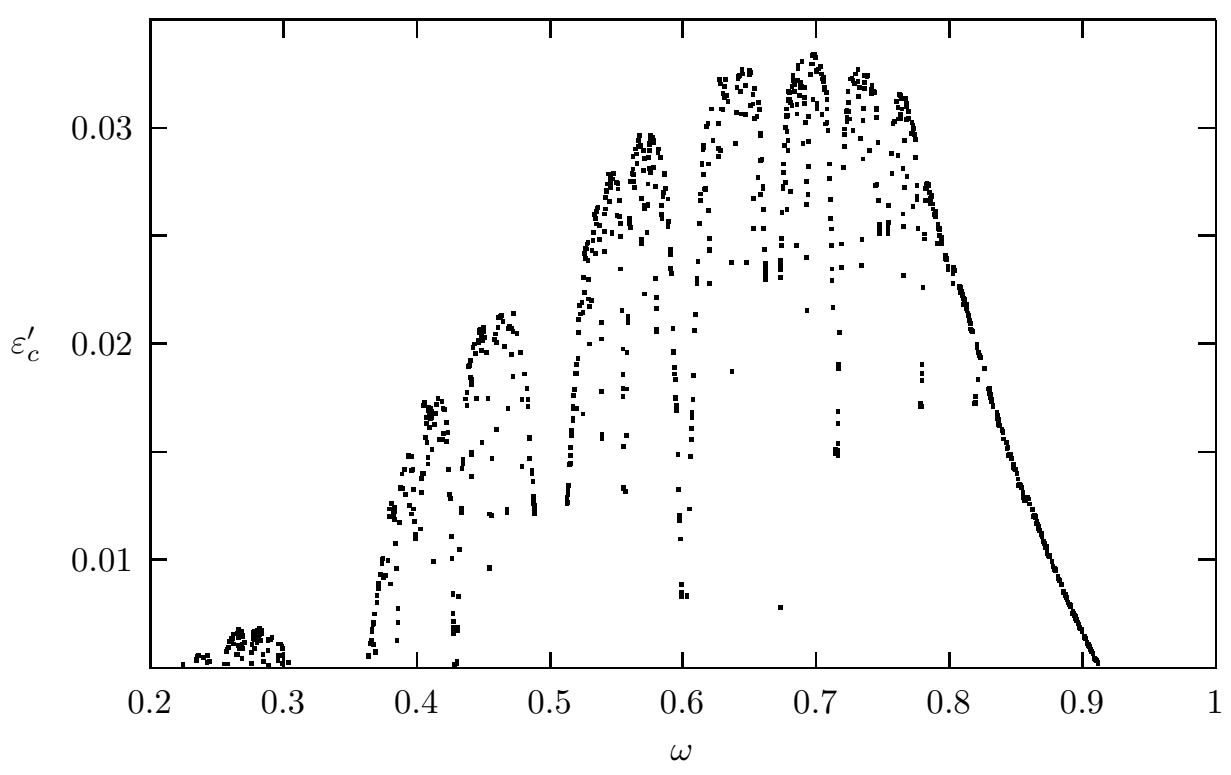

